\title{
OHENE DJAN. UN MILITANT PANAFRICANISTE À LA CONQUÊTE DE LA FIFA ?
}

\author{
Claire Nicolas, Philippe Vonnard
}

De Boeck Supérieur | «Staps »

2019/3 n 125 | pages 49 à 68

ISSN 0247-106X

ISBN 9782807393394

Article disponible en ligne à l'adresse :

https://www.cairn.info/revue-staps-2019-3-page-49.htm

Distribution électronique Cairn.info pour De Boeck Supérieur.

(C) De Boeck Supérieur. Tous droits réservés pour tous pays.

La reproduction ou représentation de cet article, notamment par photocopie, n'est autorisée que dans les limites des conditions générales d'utilisation du site ou, le cas échéant, des conditions générales de la licence souscrite par votre établissement. Toute autre reproduction ou représentation, en tout ou partie, sous quelque forme et de quelque manière que ce soit, est interdite sauf accord préalable et écrit de l'éditeur, en dehors des cas prévus par la législation en vigueur en France. Il est précisé que son stockage dans une base de données est également interdit. 


\title{
Ohene Djan. Un militant panafricaniste à la conquête de la FIFA ? Ohene Djan. A Pan-Africanist activist taking over FIFA?
}

\author{
Claire NICOLAS \\ Institut des Sciences du Sport de l'Université de Lausanne \\ Université de Lausanne \\ Quartier Unicentre \\ Bâtiment Synathlon \\ CH- 1015 Lausanne \\ claire.nicolas@unil.ch \\ Philippe VONNARD \\ Institut des Sciences du Sport de l'Université de Lausanne \\ Université de Lausanne \\ Quartier Unicentre \\ Bâtiment Synathlon \\ CH- 1015 Lausanne \\ philippe.vonnard@unil.ch
}

Résumé : En 2004, le stade national d'Accra est renommé stade Ohene Djan, ce geste devant permettre de commémorer l'action du premier directeur des sports du Ghana (1960-1966). Cependant, six ans plus tard, la municipalité d'Accra revient sur ce choix des autorités nationales, argumentant qu'il s'agit d'un stade municipal et non d'un stade national pour justifier cette décision. Si Ohene Djan fait encore parler de nos jours, c'est que sans doute que son action a largement dépassé les frontières du Ghana. En effet, nommé directeur des sports du Ghana en 1960 par Kwame Nkrumah, ce dernier a incarné durant les six ans de son mandat l'irruption d'enjeux politiques proprement africains au sein du football mondial, et a œuvré à reconfigurer des relations diplomatiques sportives jusque-là largement cantonnées à l'Europe et à l'Amérique du Sud.

Dans le cadre de cette contribution, il s'agit de penser le parcours biographique de Djan comme un point de rencontre entre les footballs - national et global - d'une part et entre une trajectoire politique et sportive d'autre part. Moins que le récit de la chronologie d'un parcours biographique rythmé par des étapes organisationnelles, politiques, personnelles et sportives, cet article considère Djan comme le nœud qui nous permet d'interroger le modèle sportif et politique de la FIFA, revendiqué comme apolitique et élitaire.

La présente recherche s'appuie sur une documentation collectée dans les riches archives de la Fédération internationale de football association (FIFA) qui a été croisée par des informations issues des fonds des Archives nationales du Ghana. Enfin, l'étude bénéficie de la lecture de journaux sportifs et généralistes ghanéens, qui permettent de mettre en lumière des éléments qui ne sont pas traités dans les documents officiels de la FIFA ou dans les archives nationales ghanéennes, mais aussi d'avoir un regard sur la présence publique de Djan.

Mots-cĹśs : Histoire, football, Ghana, FIFA, organisations internationales, Panafricanisme

Abstract: In 2004, the Accra National Stadium was renamed after Ohene Djan, who was Ghana's first director of sports from 1960 to 1966. However, in 2011, the Accra Metropolitan Assembly chose to revert to the sports arena's old name, Accra Sports Stadium, arguing that it was a city stadium and not a national stadium. It seems that Ohene Djan is still making a name for himself nowadays, 
and this is probably because his deeds went way beyond Ghana's borders. Appointed as director of sports in 1960 by Prime Minister and President Kwame Nkrumah, during his six years in this position he embodied the coming of age of Africa's political role within global football. He worked to expand diplomatic sporting ties that were, until then, largely confined to Europe and South America.

In this paper, we wish to consider Djan's biographical trajectory as a meeting point between football—national and global—on one hand and as a political and sporting trajectory on the other hand. Here, Djan's biography is less a tale of organizational, political, personal, and sporting deeds than a wealth of information that allows us to question FIFA's political and sporting model, claimed to be apolitical and elite.

This research is based on sources gathered from the archives of the Fédération internationale de football association (FIFA), alongside colonial and postcolonial archive material collected from the National Archives of Ghana and articles from sporting and generalist Ghanaian newspapers. These articles shed light on events that have no traceable trail in the archives, as well as enabling us to examine Djan's public presence.

KEYWORDs: history, football, Ghana, FIFA, international organizations, pan-Africanism

\section{INTRODUCTION}

En 2004, le stade national d'Accra est renommé stade Ohene Djan, ce geste devant permettre de commémorer l'action du premier directeur des Sports du Ghana (1960-1966). Cependant, six ans plus tard, la municipalité d'Accra revient sur ce choix des autorités nationales, argumentant qu'il s'agit d'un stade municipal et non d'un stade national pour justifier cette décision. Ces deux opérations ont été l'occasion de débats houleux, que ce soit dans la presse ou sur les réseaux sociaux ${ }^{1}$.

Si Ohene Djan fait encore parler de nos jours, c'est sans doute que l'action de cet individu a largement dépassé les frontières du Ghana. En effet, nommé directeur des Sports du Ghana en 1960 par Kwame Nkrumah, ce dernier a incarné durant les six ans de son mandat l'irruption d'enjeux politiques proprement africains au sein du football mondial et a œuvré à reconfigurer des relations diplomatiques sportives jusque-là largement cantonnées à l'Europe et à l'Amérique du Sud (Dietschy, 2013 ; Vonnard \& Quin, 2017). À la fois reflet et artisan de la répartition continentale de la Fédération internationale de Football Association (FIFA) dans les décennies 1950-1970, son positionnement résolument politique (notamment sur le cas de l'Afrique du Sud) va à l'encontre des codes sociaux et institutionnels de la Fédération internationale - et plus largement des nombreuses organisations sportives internationales - tels qu'ils ont été développés par les Européens. En d'autres termes, se focaliser sur la position de Djan permet autant de souligner les enjeux de l'internationalisation croissante de la FIFA après la Deuxième Guerre mondiale que de discuter de la "politique de l'apolitisme » (Defrance, 2000) qui est en vigueur à la Fédération internationale depuis l'entre-deux-guerres (Vonnard, 2017).

La figure d'Ohene Djan a certes été traitée à la marge de travaux généraux consacrés au football ghanéen (Bediako, 2012) ou à la place conquise par le continent africain dans la FIFA (Darby, 2000 ; Dietschy \& Kemo-Keimbou, 2008 ; Alegi, 2010), cependant sa trajectoire en tant que telle n'a pas encore fait l'objet de

1 Accra Sports Stadium is now Ohene Djan Sports Stadium, 27 novembre 2004, Ghanaweb. https://www.ghanaweb.com/ GhanaHomePage/SportsArchive/Accra-Sports-Stadium-is-now-Ohene-Djan-Sports-Stadium-70631 ; Ohene Djan Stadium renamed Accra Sports Stadium, 16 juin 2011, Ghanaweb. https://www.ghanaweb.com/GhanaHomePage/SportsArchive/ Ohene-Djan-Stadium-renamed-Accra-Sports-Stadium-211396. 
travaux spécifiques. Ce déficit d'études s'explique essentiellement pour deux raisons. D'une part, jusqu'ici, les recherches portant sur l'histoire du football ghanéen s'attellent davantage à explorer l'importation du jeu via la colonisation britannique (Darby, 2000) ou son rôle dans le projet de construction nationale porté par Kwame Nkrumah (Darby, 2013). D'autre part, les travaux sur l'élite dirigeante de la FIFA ont principalement été consacrés à ses présidents ou aux membres européens de son comité exécutif (Tomlinson, 2000 ; Dietschy, 2011 ; Bayle \& Clastres, 2018 ; Quin, Vonnard \& Jaccoud, 2019 ; Rofe, Tomlinson, 2019).

Dans le cadre de cette contribution, il s'agit de penser le parcours biographique de Djan comme un point de rencontre entre les footballs - national et global - d'une part et entre une trajectoire politique et sportive d'autre part. Moins que le récit de la chronologie d'un parcours biographique rythmé par des étapes organisationnelles, politiques, personnelles et sportives, cet article considère Djan comme le nœud qui nous permet d'interroger le modèle sportif et politique de la FIFA, revendiqué comme apolitique et élitaire. Dans les lignes qui suivent, nous proposons de suivre un fil chronologique, tout en parcourant le monde avec lui, d'Accra à Londres, Rome ou Tokyo, en passant par Addis-Abeba.

La présente recherche s'appuie sur une documentation collectée en Suisse et au Ghana. Les archives du Centre de documentation de la FIFA à Zurich donnent à voir des documents relatifs aux séances du comité exécutif et de l'assemblée générale ainsi que les dossiers de correspondance Ghana-FIFA, Confédération africaine de Football (CAF)-FIFA et du président de l'époque, Stanley Rous. Cette documentation a été croisée avec des archives (procès-verbaux de réunions, notes de service et correspondances) coloniales et postcoloniales récoltées au Ghana dans les archives nationales d'Accra (Public Records and Archives Administration Department, PRAAD), tout particulièrement celles de la Central Organisation of Sports ainsi que la correspondance d'Ohene Djan. Enfin, l'étude bénéficie de la lecture de journaux sportifs et généralistes ghanéens, qui permettent de mettre en lumière des éléments qui ne sont pas traités dans les documents officiels de la FIFA ou dans les archives nationales ghanéennes, mais aussi d'avoir un regard sur la présence publique de Djan.

\section{Un Militant ANTiCOLONial À LA PÉRIPHÉRIE DE LA GLOBALISATION DU SPORT COLONIAL (1925-1957)}

Né dans les années 1920, Ohene Djan, originaire d'un district rural, n'a pas connu le cursus classique des élites africaines anglophones (comme des études supérieures à l'étranger). Comment expliquer son parcours qui va le voir occuper une fonction clé du gouvernement ghanéen de 1960 à 1966 ainsi qu'un poste de représentant de la CAF au sein du comité exécutif de la FIFA dans la première moitié des années 1960 ? Pour comprendre cette situation, il faut tout d'abord répondre à deux questions. Premièrement, quelle est la trajectoire politique et sociale de Djan sous la domination impériale britannique, alors que le Ghana est encore la colonie de Gold Coast ? Deuxièmement, quelle est la place de ce territoire au sein de la FIFA, une organisation créée par des associations de football européennes qui, comme l'a relevé en particulier Paul Dietschy, restent largement imprégnées par une pensée eurocentrée jusque dans les années 1960 (Dietschy, 2013) ?

\subsection{Vers un investissement politique anticolonial ?}

Ohene Djan est un individu multiforme : investi en politique, homme d'affaires et administrateur sportif. Les traces archivistiques concernant sa jeunesse dessinent le portrait d'un jeune homme au plus près des mouvements historiques qui secouent la colonie britannique de Gold Coast. 
Né le 29 janvier 1925 à Aburi dans le district d'Akwapim, à une trentaine de kilomètres au nord d'Accra, Djan a suivi un cursus primaire dans l'école missionnaire locale (les missions chrétiennes européennes constituent l'essentiel du paysage scolaire colonial) avant d'intégrer une des rares écoles secondaires de Gold Coast, l'Accra Academy. Elle l'accueille jusqu'en 1943, date à laquelle Djan finit sa scolarité (PRAAD, Report of the Manyo-Plange (Assets) Commission, 1969). Originaire d'un district rural et d'une famille de négociants - son père travaille dans le commerce du cacao -, il a aussi un positionnement privilégié au sein des élites locales puisqu'il fait partie de la famille élargie de Sa Majesté (Nana) Osae Djan II, qui occupe le prestigieux siège (stool) d'Aburi (Daily Graphic, 16 juillet 1952, p. 3). Son prénom, Ohene, traduit d'ailleurs bien cette filiation (Duschesne \& Guedj, 2005, p. 139). Enfin, relevons que, de la fin de ses études à 1949, il est employé au sein du gouvernement colonial comme clerc, avant de rejoindre le commerce familial (Vieta, 1999). Cette proximité avec les autorités locales (administration coloniale comme chefferie, les deux étant rarement antinomiques) lui offre une place privilégiée pour se positionner dans la vie politique de la colonie.

$\mathrm{Au}$ regard de ses origines sociales et du début de son parcours professionnel, nous postulons que Djan se rapproche des trajectoires masculines d'Akrakyefor (employé ou commis en akan, dérivé du mot anglais clerk) analysées par Stephen Miescher dans le Ghana colonial des années 1930-1940. Il a en effet les mêmes caractéristiques sociales que ces intermédiaires du gouvernement colonial, comme l'alphabétisation ou l'ambivalence politique. Si Djan a un statut privilégié indéniable au regard de l'ensemble de la population de Gold Coast, pour autant il ne fait «ni partie des élites anciennement établies en tant que "chefs traditionnels" - en charge de l'administration locale dans le cadre du gouvernement de l'indirect rule - ni de l'intelligentsia [africaine] très instruite et financièrement à l'aise » vivant dans les villes côtières du pays (Miescher, 2005, pp. 84-85).

En cette fin des années 1940, le contexte sociopolitique de la Gold Coast est particulièrement violent. Alors que les fluctuations du cours du cacao et la récession économique pèsent sur la population colonisée, manifestations, grèves et boycotts se multiplient. Or la réaction du gouvernement colonial est extrême : emprisonnements, meurtres, répression policière et militaire. Ces heurts sont concomitants avec la montée en puissance des revendications nationalistes des partis indépendantistes africains. Fondée en 1947, l'United Gold Coast Convention (UGCC) rassemble les élites africaines (tant des chefs « traditionnels » que l'intelligentsia urbaine - universitaires, avocats, etc.) autour d'un projet nationaliste. Kwame Nkrumah, revenu du Royaume-Uni en 1948 - où il a notamment participé à d'importants échanges au sein du mouvement panafricaniste, comme la Conférence de Manchester de 1945 (Bouamama, 2014) -, milite au sein de l'UGCC avant de rompre et de fonder le Convention People's Party (CPP). Ce parti s'inscrit dans une perspective plus radicale que l'UGCC. Plus que le nationalisme, Nkrumah revendique un idéal panafricain (unir tous les Africains), anti-impérialiste (contre la domination coloniale occidentale) et socialiste (en s'inspirant des modèles soviétiques et chinois). Le CPP propose de renverser les hiérarchies sociales et devient progressivement le « premier parti nationaliste de masse » de la colonie (Allman, 1993, p. 139), des jeunes, des femmes, des ruraux ou encore des Akrakyefor y adhérant en nombre. Djan, comme de nombreux autres jeunes gens de la colonie, est donc séduit par la vision de Nkrumah et s'engage à ses côtés.

Alors que les mouvements indépendantistes ébranlent l'Empire britannique - déjà amputé, rappelons-le, du sous-continent indien et de ses possessions au Moyen-Orient - aux quatre coins du monde, les revendications du CPP débouchent finalement, en février 1951, sur 
la tenue d'élections législatives qui doivent permettre d'élire les membres du premier Parlement africain. Le CPP remporte les élections et Nkrumah devient Premier ministre de Gold Coast. Ohene Djan bénéficie de ce changement politique et obtient le poste de député de la circonscription d'Akwapim / New Juaben. Mais l'aura du CPP n'est pas hégémonique. Durant les mois qui suivent, ses dirigeants sont contestés par des organisations politiques concurrentes régionalistes (Allman, 1993), voire par la branche communiste du parti, qui les accuse de délaisser le peuple au profit de manœuvres politiciennes (Ahlman, 2017, pp. 121-122). Lors des nouvelles élections générales de 1954, Djan ne reprend pas son mandat. Plus précisément, il apparaît qu'il fait partie d'un lot de parlementaires écartés du pouvoir pour des questions de malversations financières (Austin, 1966).

Les fragments collectés autour de la jeunesse de Djan nous permettent de dresser le portrait d'un édile africain bien particulier. Djan se constitue un capital immobilier dans la région d'Accra (PRAAD, Report of the ManyoPlange (Assets) Commission, 1969) et est proche de Nkrumah - dont l'aura ne cesse de croître - comme des autorités politiques " traditionnelles ». Cela nous donne un aperçu intéressant de la position sociale du natif d'Aburi. Non seulement il dispose d'un capital économique, politique et relationnel de plus en plus important, mais ces premières années dessinent un homme habitué à naviguer entre différents milieux sociaux : le fonctionnariat colonial britannique, le Parlement de Gold Coast, le monde des affaires et les chefferies.

\subsection{Devenir un dirigeant sportif}

Parallèlement à ses activités politiques et commerciales, Djan commence à être actif dans le domaine sportif. À Accra, en 1953, il organise des combats de boxe payants (PRAAD, Procès-verbal de la réunion du Gold Coast Amateur Sports Councildu 27 octobre 1953) et s'investit pour améliorer les infrastructures de spectacle de la capitale (PRAAD, Rapport sur le King George V Memorial Hall, 21 juillet 1953). Au-delà de son ancrage politique, le jeune homme s'inscrit dans le renouveau des pratiques de loisirs urbains, plébiscitées par les jeunes gens d'Accra, entre voitures (il acquiert une Mercedes !), musique highlife, dancings, football, boxe et cinéma (Plageman, 2013).

Ohene Djan est donc un acteur multiforme. Toutefois, notons tout de même que, comparativement à son association précoce à la vie politique et économique de Gold Coast, il n'intègre que tardivement les organisations sportives. En effet, dans les années 1950, la gestion du sport reste la prérogative d'hommes africains plus âgés, diplômés au Royaume-Uni ou dans des écoles supérieures locales et insérés dans la clubbability sportive d'Accra. Ces hommes font partie des cercles sociaux et culturels des villes côtières étudiés par Stephanie Newell (2006). Ce sont eux, sans Djan, qui initient la première phase d'internationalisation du football ghanéen. Ils ont institutionnalisé les premiers clubs et associations sportives régionales à partir des années 1920 (athlétisme, cricket, hockey, tennis, football, etc.).

Sport emprunté aux colons britanniques, le football est pratiqué depuis les villes de la côte atlantique jusqu'aux mines d'or de l'Ashanti, tant par les classes populaires que par la bourgeoisie urbaine où a été fondé un nombre croissant de clubs, et ce depuis le début du XX siècle (Darby, 2000), à l'instar de ce qui se passe dans bon nombre de colonies anglaises (Alegi, 2010). Le ballon rond devient peu à peu une pratique culturelle majeure pour les jeunes hommes de la colonie. Après la Deuxième Guerre mondiale, les élites urbaines nationalistes qui dirigent les associations sportives unifient les multiples clubs du pays au sein de l'United Gold Coast Football Association (UGCFA) en 1950 (PRAAD, Correspondances de l'Accra Football League, de l'Amateur Football Association et de la Ga Football Association, 1920-1940).

Conjointement, la colonie commence à être représentée à l'échelle internationale 
et intègre la FIFA - les colonies britanniques ayant obtenu le droit de la Football Association (Fédération anglaise de football) de disposer de leur autonomie. À ce moment, la Fédération internationale connât une internationalisation croissante qui va d'ailleurs conduire à une progressive décentralisation de sa structure (Charroin \& Waquet, 2008 ; Vonnard, 2018)2. Tout d'abord, la colonie intègre la FIFA au titre d'une association locale (les représentants proviennent d'un club d'Accra) en 1948, puis de l'UGCFA en 1952, présidée par Richard Akwei (FIFA, Procès-verbal de l'assemblée générale de la FIFA des 27-28 juillet 1948 et lettre de Richard Akwei au secrétaire général de la FIFA, 21 août 1954). Pour les dirigeants ghanéens, l'adhésion à la Fédération internationale est un jalon essentiel en vue de créer des contacts et de disputer des rencontres avec d'autres pays et in fine d'améliorer le niveau général du football. Si Djan joue certainement au football pendant cette période (notamment en raison de sa scolarisation en école secondaire), force est de constater qu'il ne participe pas à cette reconfiguration de l'organisation du football en Gold Coast. Son investissement dans la vie sportive s'opère d'abord en dehors du champ footballistique, au sein du Gold Coast Amateur Sports Council (Conseil du sport). Cette structure parapluie associe l'ensemble des organisations sportives à partir de son institution en 1952 par l'administration coloniale.

C'est en 1957, l'année même où le Ghana devient indépendant, qu'il est propulsé à la tête de ce qui devient par la même occasion la Ghana Football Association (GFA). Djan semble moins s'appuyer sur son expérience en matière sportive que sur son capital politique et sa proximité avec Nkrumah pour se faire élire et diriger l'organisation principale du football ghanéen.

\section{2. "Osagyefo's sportsman \#, à la conquête des organisations sportives internationales (1957-1962)}

En 1957, la Gold Coast, conduite par son charismatique Premier ministre, obtient son indépendance. Le pays, qui prend le nom de Ghana, entame dès lors une révolution autant politique que culturelle et, trois ans plus tard, prend le statut d'une République, Kwame Nkrumah devenant le premier Président du pays. Ce faisant, le Ghana accélère son internationalisation sportive, accélération portée par Ohene Djan lui-même. Lorsque ce dernier accède au comité exécutif de la FIFA en 1962, il a 37 ans et est un membre convaincu de l'administration nkrumahiste. Il se profile rapidement comme un acteur clé du football tant à l'échelle continentale que mondiale.

\subsection{Prendre en main le football ghanéen}

Le $1^{\text {er }}$ juillet 1957 , le jour même de la proclamation de la première République du Ghana, Ohene Djan est nommé au poste de directeur des Sports. À ce titre, il est chargé de réformer et de développer le sport ghanéen selon une ligne «nkrumahiste $»^{3}$. Ce choix peut paraître surprenant : après tout, il n'a pas l'expérience de dirigeant des leaders associatifs coloniaux, en place depuis une vingtaine d'années. Nous faisons l'hypothèse que la nomination de Djan participe de la volonté de rupture de Nkrumah. Les dirigeants sportifs qui le précèdent sont plus âgés, et éventuellement membres de partis nationalistes concurrents du CPP. Ils font donc partie d'une intelligentsia qui adhère aux valeurs culturelles britanniques. Par conséquent, le choix d'un jeune Akrakyefos fidèle au CPP à la tête du mouvement sportif ghanéen permettrait d'instaurer une rupture idéologique et symbolique avec la période coloniale.

\footnotetext{
2 Cette décentralisation se concrétise par la réforme des statuts de la FIFA en 1953 qui conduit à la création de groupements continentaux en Europe, Asie et en Afrique, sur le modèle de la Confédération sud-américaine, créée en 1915-1916.

3 Comme signalé par Jeffrey Ahlman, bon nombre d'archives liées au CPP ont été détruites lors du coup d'État, laissant peu de traces pour qui s'intéresse au rôle précis joué par Nkrumah dans le domaine sportif (Ahlman, 2017, p. 23).
} 
Le modèle sportif proposé par Djan et Nkrumah repose sur une centralisation des organisations sportives au sein de l'État. C'est pourquoi ils dissolvent le Conseil du sport, qu'ils considèrent comme une relique coloniale et fondent la Central Organisation of Sports qui s'inspire explicitement du modèle soviétique (PRAAD, Réponse d'Ohene Djan à la question parlementaire adressée au ministère de l'Éducation, 17 septembre 1964). L'URSS constitue alors un modèle regardé d'un bon œil par le Président ghanéen (Parks, 2014 ; Nicolas, 2017). Djan nomme ainsi les directeurs régionaux chargés de développer le sport en province et centralise le budget et la maind'œuvre sous sa direction personnelle, au sein même de l'administration publique.

Dans l'ensemble, le directeur des Sports s'octroie un pouvoir personnel important sur les activités sportives en général - le football en particulier - et n'hésite pas à revendiquer officiellement une gestion du sport inspirée du socialisme soviétique. Des liens étroits se tissent d'ailleurs entre le Ghana et l'URSS (Parks, 2014) - dans le sillage d'un accord culturel signé entre les deux pays en 1961 -, qui se concrétisent par l'invitation d'entrâ̂neurs et d'équipes soviétiques au Ghana et le départ d'athlètes en URSS pour des séjours de formation. Conjointement, Djan assume l'aspect autoritaire de sa politique, écrivant en 1964 : « Le nouveau modèle n'a pas de place pour une approche nonchalante vis-à-vis du développement des sports, par des comités "au bon vouloir de chacun", qui étaient la marque de fabrique des sports de Gold Coast avant l'indépendance " (PRAAD, Réponse d'Ohene Djan à la question parlementaire adressée au ministère de l'Éducation, 17 septembre 1964). Cet autoritarisme du directeur des Sports se déploie d'autant plus librement que le gouvernement de Nkrumah devient, au fil des ans, de plus en plus coercitif (Ahlman, 2017, p. 150). Dans le cadre de cette politique de "nkrumahisation " du pays, le sport roi que constitue le football est un enjeu de taille pour gagner les corps et les cœurs à la cause du Président (Darby, 2013).

Le sport ghanéen est alors incarné par la figure de Ohene Djan. En outre, son enrichissement personnel pendant son séjour à la tête de la direction des Sports soulève les fantasmes, mais crée aussi des oppositions. De fait, il mène à cette période un train de vie luxueux. En tant que notable, il a la charge de sa famille immédiate et élargie : sa mère désormais veuve (PRAAD, Report of the Manyo-Plange (Assets) Commission, 1969, p. 4). Au-delà de ce train de vie qui est parfois brocardé par ses concitoyens, en 1963, Djan se fait également accuser de népotisme au Parlement. On lui reproche par exemple de n'engager que son entourage au sein de la direction des Sports (PRAAD, Question parlementaire adressée au ministère de l'Éducation, 22 mars 1963). Défendu par le ministre de l'Éducation, il est innocenté, chiffres à l'appui. Pour autant, l'accusation n'était pas sans fondements. Son propre frère dirige la Fédération des sports scolaires et universitaires, son cousin est employé comme comptable et un/une autre membre de sa famille comme secrétaire (PRAAD, Procèsverbal de la réunion de la Schools and Colleges Sports Federation de Volta, 27 janvier 1962).

Le jeune politicien méconnu des années 1950, dépendant financièrement de ses aînés, est devenu un homme aisé, un acteur clé de l'appareil d'État et de la vie publique ghanéenne. Il est cité hebdomadairement dans le Daily Graphic, le journal national, pour commenter sa politique sportive et les résultats sportifs internationaux. La presse le surnomme le «sport-czar » ou « Osagyefo sportsman ", lorsqu'elle reprend le surnom élogieux attribué à Nkrumah. Dans les photographies de presse, il apparaît presque exclusivement drapé dans un pagne kente, ce symbole du pouvoir akan ${ }^{4}$, que ce soit au Ghana ou lors

4 Les pagnes kente sont des tissus de coton composés de bandes de tissu de plusieurs couleurs entrelacées. Ils sont typiques des peuples akans. Ils sont portés par des rois (hommes ou femmes) où lors de circonstances de prestige (mariages, enterrements). Les pagnes 
de matchs de l'équipe nationale ainsi que durant leurs séjours à l'étranger. Ce faisant, il joue des mêmes codes vestimentaires que Nkrumah lui-même (Monfils, 1977, p. 315) et participe à incarner le projet politique panafricain : tourné vers le monde extérieur tout en restant attaché à ses racines ghanéennes.

Ohene Djan conduit une politique de centralisation des activités sportives, centrée autour de sa personne et d'un réseau de fidèles, tout en contrôlant étroitement la médiatisation des sports. En 1960, il fonde l'Overseas and Olympics Games Committtee (OOGC), qui lui donne la mainmise sur l'ensemble des relations internationales sportives, celles-ci étant de trop grande importance pour être laissées aux mains de qui que ce soit d'autre. À ce titre, il écarte définitivement des personnalités influentes du sport ghanéen des années 19401950, comme que Richard Akwei (PRAAD, Rapport de la commission Kwaw-Sanzy, 1960). Non sans débats houleux, le comité olympique privé est finalement rattaché à la direction des Sports et se situe dès lors au cœur de l'appareil d'État (PRAAD, Procès-verbal de la réunion du comité exécutif du Ghana Amateur Sports Council, 26 mai 1960). Devenu le dépositaire des relations sportives internationales ghanéennes, au début des années 1960, Djan fait une irruption remarquée dans le champ institutionnel de la FIFA.

\subsection{Représenter le continent africain à la FIFA ?}

La première participation d'Ohene Djan à l'assemblée générale de la FIFA a lieu dès 1960 (FIFA, Procès-verbal de l'assemblée générale de la CAF du 21 août 1960). Il représente alors un pays qui prend de plus en plus d'importance dans le football africain. Les Black Stars - l'équipe nationale ghanéenne - deviennent un élément essentiel de la scène footballistique africaine, allant jusqu'à remporter la Coupe
d'Afrique des Nations en 1963 et 1965. De même, en janvier 1965, la capitale du Ghana accueille la finale de la première édition de la Coupe des clubs champions africains. Il faut comprendre cette ambition dans le cadre de la volonté du gouvernement ghanéen d'investir les scènes internationale et continentale ; en témoigne le rôle de Nkrumah dans la fondation de l'Organisation de l'Unité africaine (OUA) ou ses prises de position remarquées en faveur du Tiers-Monde (Bouamama, 2014).

Mais, l'investissement dans le football international passe désormais également par l'intermédiaire des nouvelles organisations continentales, créées durant la seconde partie des années 1950 (Vonnard, 2018). Ainsi, pour assumer une position de leader des nations africaines, il est nécessaire pour Djan de bien figurer au sein de la Confédération africaine de football, fondée entre 1956 et 1957 en 1956 autour de l'Éthiopie, de la République Arabe Unie (RAU) ${ }^{5}$ et du Soudan (FIFA, Note sur l'assemblée générale de la CAF du 3 mai 1959). Signe de cette position de plus en plus importante prise par le Ghana dans le football continental, les dirigeants de la CAF semblent souscrire à l'idée qu'un représentant de ce pays soit dorénavant présent au sein du comité exécutif de l'organisation continentale. En effet, en février 1961, une réunion extraordinaire de la CAF décide qu'un Ghanéen devra intégrer la nouvelle composition de son comité exécutif (FIFA, Procès-verbal de l'assemblée extraordinaire de la CAF des 16 et 17 février 1961). Un an plus tard, lors d'une nouvelle assemblée extraordinaire de la CAF organisée à Addis-Abeba, la place du Ghana est confortée. Djan ne se déplace pas personnellement, mais délègue un fonctionnaire de l'OOGC. Au moment de l'élection des deux représentants africains qui siégeront dans le comité exécutif de la FIFA, le délégué ghanéen n’hésite pas à souligner que cette question est la plus importante de

se différencient selon leurs motifs, nommés d'après des événements historiques. Ils sont portés au Togo, en Côte d'Ivoire et au Ghana. 5 À cette période, l'Égypte a fusionné avec la Syrie (accompagnée un temps durant par le Yémen) pour créer la République Arabe Unie (RAU). Cette union existe formellement de 1958 à 1961, l’Égypte conservant ensuite ce nom jusqu'en 1971. 
l'ordre du jour et ajoute que le choix devrait se porter sur des dirigeants disposant de capacités d'« initiative [...] et [d']une large expérience dans le domaine du travail » (FIFA, Procès-verbal de l'assemblée extraordinaire de la CAF des 16 et 17 janvier 1962). De plus, il ajoute qu'un des délégués doit être capable de représenter les associations de l'Afrique de l'Ouest. De fait, les qualités décrites par le dirigeant ghanéen préparent habilement le terrain à la candidature de son directeur des Sports. Alors que l'Égyptien Mostafa est élu au poste de « vice-président ", Djan obtient la place de «membre " d'un vote (quatre contre trois). Son élection a lieu au détriment d'un dirigeant qui a pourtant été très actif au sein de la CAF depuis sa création, l'Éthiopien Ydnekatchew Tessema.

Comment expliquer l'acceptation rapide de Djan en tant que leader de la CAF ? Outre l'importance progressive du Ghana, son arrivée incarne plus largement la montée en puissance proprement footballistique (et non pas seulement administrative) de l'Afrique occidentale et subsaharienne, qui rivalise désormais sur le terrain avec les champions du Nord-Est du continent. De fait, la présence du dirigeant ghanéen modifie l'échiquier politique de la CAF, et en particulier contribue à l'émergence d'un pôle régional ouest-africain devant contrebalancer le leadership égypto-éthiopien (Dietschy \& Kemo-Keimbou, 2008 , p. 159). Mais, nous faisons également l'hypothèse que le choix de Djan révèle l'aura du panafricanisme incarné par Nkrumah chez de nombreux membres des élites sportives du continent et, en ce sens, l'émergence d'une volonté plus marquée de défendre les intérêts des associations africaines au sein du comité exécutif de la FIFA (Darby, 2002, p. 36). En compagnie de Mostafa et de Tessema (qui reste actif au sein de la CAF), Djan est désormais l'un des principaux leaders du football africain et c'est presque logiquement qu'il est dans la foulée élu comme vice-président de la CAF
(FIFA, Procès-verbal de l'assemblée extraordinaire de la CAF des 23 et 24 janvier 1963).

C'est donc au titre de représentant de l'organisation du football africain que Djan intègre le comité exécutif de la FIFA. Au premier abord, son action semble globalement limitée. Nous avançons que cela s'explique largement en raison de son profil atypique en comparaison des dirigeants européens qui œuvrent dans le comité de la FIFA. Plus jeune d'une trentaine d'années que ses collègues, son expérience en matière d'administration sportive apparaît comme restreinte et pèse peu contre des dirigeants de l'envergure du président Stanley Rous. Ce dernier dirige la FIFA depuis 1961 et était déjà secrétaire général de la puissante Football Association lorsque Djan... n'avait que six ans! Plus encore, sa couleur de peau et son origine subsaharienne ne sont certainement pas sans influence sur l'atypisme de son profil au sein d'une FIFA qui reste très blanche et conservatrice, dominée par des dirigeants issus des anciennes métropoles coloniales elles-mêmes. De plus, ces dirigeants européens partagent une vision proche du monde et sont à placer sur l'échiquier politique européen entre les libéraux conservateurs et les démocrates-chrétiens (Vonnard, 2017).

Enfin, Djan découvre le fonctionnement d'une organisation internationale qui a pris une ampleur sans précédent dans les récentes décennies. S'il est un homme politique expérimenté en Gold Coast et a pu aisément gagner sa position à la CAF, qu'en sera-t-il au cœur de la plus grande fédération sportive du monde? Comment va-t-il s'intégrer aux usages sociaux de la FIFA, alors que ceux-ci répondent à des codes précis ? Ceux-ci sont partagés par la grande majorité des dirigeants européens qui prennent part aux activités de la FIFA : comme la maîtrise de plusieurs langues européennes, une manière diplomatique de conduire les débats publics, la rhétorique de l'apolitisme ainsi qu'une pratique du pouvoir fondé sur les 
discussions informelles, en marge des réunions officielles (Vonnard, 2018).

La position sociale du dirigeant ghanéen, qui s'ajoute au fait qu'il provient d'un continent encore considéré comme peu influent en termes de football par ses collègues européens (Darby, 2019), explique sans doute la position que nous pouvons qualifier de secondaire qu'il occupe au sein du comité exécutif. Même si, de 1962 à 1966, il est présent à toutes les séances (environ deux par années, cette fréquence témoignant de l'importance accordée à ces déplacements pour le gouvernement ghanéen qui finance ses voyages en Europe), force est de constater qu'il ne siège dans aucune des principales commissions permanentes (finances, Coupe du Monde) de la FIFA. De même, lorsque des discussions ont lieu sur la composition des différentes commissions de la future Coupe du Monde de 1966, il ne se voit confier qu'un seul poste (alors que les dirigeants plus expérimentés cumulent plusieurs fonctions), et qui plus est dans une commission qui ne compte pas parmi les plus prestigieuses : la commission disciplinaire (FIFA, Procès-verbal du comité exécutif de la FIFA du 23 janvier 1963).

En octobre 1964, conscients qu'il devient nécessaire d'accorder plus de place aux dirigeants qui ne participent pas aux réunions du comité d'urgence, les principaux dirigeants de la FIFA (sous l'impulsion du président Stanley Rous) décident de faire quelques ajustements. Désormais, un dirigeant comme Djan aura la possibilité d'assister à quelques séances du comité d'urgence, pour autant qu'il soit disponible et surtout que le sujet traité touche de près ses prérogatives, à comprendre les questions relatives au football africain. En effet, et contrairement à ses collègues européens ou sud-américains, le Ghanéen ne se voit pas accorder le statut de dirigeant pouvant " traiter des affaires urgentes » (FIFA, Procès-verbal du comité exécutif de la FIFA du 17 octobre 1964).

Cette position secondaire au sein du comité exécutif de la FIFA peut s'expliquer par sa trajectoire sociale atypique et sa position subalterne, face à des acteurs européens conservateurs qui peinent à prendre la mesure de la décolonisation. Mais ne s'explique-telle pas aussi par sa position politique subversive ? En effet, au regard des revendications panafricaines de son gouvernement, Djan risque de déranger l'ordre établi de la FIFA. Et il ne se prive pas de le faire lorsqu'il s'agit d'aborder des sujets sensibles dont les enjeux dépassent largement le cadre de la Fédération internationale.

\section{DÉCOLONISER LA FIFA (1962-1966) ?}

L'arrivée en force des pays africains au début de la décennie remet en question la géopolitique interne de la FIFA et pose des problèmes d'ordre "structurel ». Dans le livre du centenaire de la FIFA Eisenberg, Lanfranchi, Mason et Wahl notent : "L'anonymat croissant et la multiplicité des langues contribuent à une défiance réciproque, déjà attisée par la Guerre froide » (2004, P. 286). En outre, cette situation remet en question l'apolitisme conservateur qui présidait au sein de l'organisation (Vonnard, 2017). En effet, pour les dirigeants européens, il est essentiel de ne pas mélanger le football et la politique. Cette posture est, dans les faits, souvent bafouée tant en raison de l'investissement croissant des États dans le football international depuis l'entredeux-guerres (Macon, 2008) que de l'activité politique des dirigeants de la Fédération (Vonnard, 2018). Après la Deuxième Guerre mondiale, de nouveaux acteurs prennent de l'importance au sein de la FIFA - situation qui se perçoit également dans de multiples autres organisations internationales (Louis, 2017). Notamment, des dirigeants sportifs issus des 
anciennes colonies négocient leur entrée dans la FIFA. Au fur et à mesure de leur participation aux activités de la Fédération internationale, Djan, Mostafa ou Tessema s'approprient les codes de ses principaux dirigeants. Par exemple, comme le montrent les correspondances régulières de Djan avec les membres de la CAF et de la FIFA, il initie des rencontres informelles en petit comité, invitant à dîner ses collègues du comité exécutif dans le but de continuer les discussions de manière informelle. Pour justifier son mode d'action auprès du gouvernement ghanéen, il fonde ses arguments sur les habitudes qui existent au sein du comité exécutif (PRAAD, Demande de remboursement auprès de la Banque nationale du Ghana, 22 décembre 1965). Son comportement en tant que représentant ghanéen à la FIFA semble donc relever d'un décodage des normes de comportement propres aux dirigeants sportifs internationaux ${ }^{6}$.

Cependant, leur action ouvre également la brèche vers une action politique subversive. Les dirigeants européens de la FIFA, de leur côté, tiennent fermement à la rhétorique de l'apolitisme conservateur qui doit permettre de maintenir une cohésion interne, mais surtout de renforcer le pouvoir de l'organisation dans l'administration mondiale du football (Vonnard, 2018). Ce faisant, Djan et ses collègues provoquent des points de tension dans l'organisation, notamment en orchestrant le boycott de la Coupe du Monde de 1966 et en luttant pour l'exclusion de l'Afrique du Sud de la FIFA.

\subsection{Faire face à l'hégémonie européenne}

Quand Ohene Djan entre au comité exécutif de la FIFA en 1962, il participe à l'émergence d'un "bloc " africain au sein de la Fédération internationale. Au cours de la première moitié des années 1960, le nombre de pays africains membres de la FIFA s'accroît constamment, suivant en cela la chronologie de la décolonisation qui voit les Africains démanteler les empires britanniques et français. Sous cet angle, adhérer à la FIFA va de pair avec l'affiliation à d'autres organismes internationaux, les Nations unies en premier chef (Darby, 2002). Dès lors, la lecture des procès-verbaux des assemblées générales nous permet de signaler que s'ils n'étaient que six pays africains à envoyer des délégués lors de l'assemblée générale de la FIFA de 1960, ils sont neuf en 1962 puis vingt-quatre, deux ans plus tard. Le chiffre commence à être conséquent, car en 1964 les associations africaines représentent dorénavant $25 \%$ des délégués présents. À titre de comparaison, cette même année, vingthuit associations nationales européennes participent à l'assemblée générale. Or, en raison de la division Est-Ouest produite par la Guerre froide - à laquelle s'ajoutent parfois des visions différentes entre les associations européennes sur la politique footballistique que doit mener la FIFA -, les Européens ne constituent pas un bloc totalement uni (Mittag, 2015). Cette situation limite leur influence : la Fédération internationale étant régie par la loi d'une association est égale à un vote, les pays africains - s'ils s'unissent - constituent désormais une force capable de porter la voix de l'Afrique au sein de l'organisation. Pour arriver à un tel résultat, les représentants de la CAF s'associent parfois à leurs collègues asiatiques pour s'opposer aux membres européens de la FIFA et promouvoir davantage la place de leur continent au sein de l'organisation.

Lors de l'assemblée générale extraordinaire de 1961, Ohene Djan appuie ainsi la demande du délégué malais, Lim Kee Siong, qui consiste à obtenir une place de vice-président et de deux membres supplémentaires pour l'Asie au sein du comité exécutif. Bien que celle-ci soit refusée après un vote serré, un an plus tard, Djan et ses collègues obtiennent une mesure intermédiaire. En effet, en 1962, de nouveaux statuts de la Fédération internationale sont

6 On peut ainsi dresser un parallèle avec le travail de Sabine Dullin. Elle décrypte le décodage des normes de la diplomatie occidentale par les diplomates soviétiques dans les années 1930 (Dullin, 2001). 
promulgués. Or ceux-ci attribuent respectivement une deuxième place aux continents africain et asiatique dans le comité exécutif (FIFA, article 18, alinéa 3, Statuts de la FIFA, 1962). Ce pas en avant, même mesuré, n'est pas du goût des représentants ouest-européens. Ainsi, en octobre 1963, le président de l'Union des associations européennes de football (UEFA) - fondée en 1954 - le Suisse Gustave Wiederkehr, demande la mise sur pied d'une commission d'étude en vue de réformer une nouvelle fois les statuts de la FIFA (FIFA, Procès-verbal de la séance du comité exécutif de la FIFA du 21 octobre 1963). L'UEFA veut abolir la règle de l'égalité des voix entre les associations nationales en la remplaçant par une règle proportionnelle, démarche qui favoriserait les grandes associations nationales, à savoir les associations européennes. Sa tentative se solde toutefois par un échec, notamment en raison de l'opposition de membres du bloc européen : les associations des pays communistes.

Mais c'est surtout à partir de 1964, lors de l'assemblée générale que Djan et ses collègues extra-européens obtiennent de véritables avancées. Réunis lors d'une assemblée plénière qui rassemble un nombre inédit de pays africains, les délégués se prononcent en grande majorité pour renforcer la continentalisation de l'organisation. Un plan est dès lors mis en place en vue de créer une répartition continentale des futures compétitions de la FIFA et de l'assemblée générale, tandis que des comités consultatifs entre la FIFA et les confédérations continentales sont créés afin d'améliorer les échanges ${ }^{7}$. De fait, les confédérations continentales extra-européennes obtiennent davantage d'espace pour exprimer leur point de vue. Djan, en compagnie de l'Égyptien Fahmy et de l'Éthiopien Tessema, en profite pour tenter (sans succès) de renforcer la position des pays africains en demandant l'obtention de quatre places pour le continent africain au sein du comité exécutif de la Fédération internationale
(FIFA, Procès-verbal de l'assemblée générale de la FIFA du 8 octobre 1964).

Durant son mandat au sein de l'organe directeur de la FIFA, Djan participe activement à ces discussions qui s'opèrent dans un contexte d'opposition entre l'UEFA et les autres confédérations continentales. La tension monte en 1964, avec une décision prise par les associations africaines : le boycott de la Coupe du Monde de 1966 qui doit se dérouler en Angleterre. Jusqu'ici, jamais l'épreuve phare de la Fédération internationale n'avait connu ce type de situation, ce qui fait dire à Stanley Rous dans son allocution qu'il « est choqué » par celle-ci et qu'il serait « très déçu si cette information devait se confirmer " (FIFA, Procès-verbal de l'assemblée générale de la FIFA du 8 octobre 1964).

Le débat s'enflamme autour du nombre de places attribuées pour les tours préliminaires. Cinquante et un pays sont inscrits aux épreuves de qualifications qui doivent sélectionner les quatorze finalistes qui rejoindront les deux pays déjà qualifiés, à savoir l'Angleterre (le pays organisateur) et le Brésil (vainqueur de l'édition précédente). Afin de les déterminer, et en suivant le procédé qui a été utilisé lors des épreuves précédentes, des zones géographiques sont établies. La répartition finale décidée par la FIFA est la suivante :

-dix places sont attribuées à la zone Europe ; -quatre places à la zone Amérique du Sud ; -une place pour l'Amérique du Nord et centrale ;

-une place à la zone Asie et Afrique

Cette disproportion est intolérable pour Djan qui prend la tête de la fronde aux côtés de Tessema (Dietschy, 2013, p. 295). Pour le dirigeant ghanéen, il est tout bonnement inadmissible que son continent ne dispose que d'une place dans le tour final, et ce d'autant plus que le qualifié africain doit encore disputer cette place au vainqueur de la zone asiatique.

Lors de l'assemblée générale de la CAF de juillet 1964 叉 et alors que l'organisation

7 Sur le modèle de la commission FIFA-UEFA mise en place en 1959. 
connait une première phase de structuration (Rofe, Tomlinson, 2019) \ les délégués réaffirment leur volonté de discuter de cette décision et soumettent le " problème à tous ceux qui ont à cœur, tant en Afrique qu'ailleurs, l'objectif de faire du Championnat du monde une véritable manifestation mondiale, loin de tout exclusivisme » (PRAAD, Lettre de Mourad Fahmy aux membres du comité exécutif de la FIFA et aux associations nationales affiliées, 10 août 1964). Par ailleurs, ils accusent le comité d'organisation de la Coupe du Monde 1966 d'employer des arguments fallacieux pour justifier ce groupement. Outre la volonté d'obtenir davantage de reconnaissance, Djan et ses collègues se plaignent également du fait que la situation ne fasse porter un coût démesuré pour les associations africaines et asiatiques, peu dotées financièrement, pour un résultat très incertain. Au contraire, les chances des riches associations européennes d'atteindre les phases finales sont bien plus favorables.

Toutefois, bien que le secrétaire général de la FIFA lui-même (le Suisse Helmut Käser) trouve " raisonnables » certains arguments des dirigeants africains, il indique que les décisions prises par la FIFA sont irrévocables (FIFA, Lettre d'Helmut Käser à Stanley Rous, non datée, août 1964). En conséquence, les 15 fédérations africaines inscrites déclarent successivement forfait pour la Coupe du Monde ${ }^{8}$. Celles-ci sont sanctionnées d'une amende de 5000 francs suisses chacune, somme importante pour ces jeunes organisations (Darby, 2002, p. 52). Cette sanction s'explique car, selon les statuts de la FIFA, ses associations membres ont le devoir de participer à ses activités. En outre, le comité exécutif de la Fédération internationale estime que « le prestige du Championnat du Monde [a été] menacé » (FIFA, Procès-verbal de la séance du comité exécutif de la FIFA du 5 janvier 1966).

La lutte pour l'obtention de places au sein du comité exécutif entre 1961 et 1964 puis le boycott des associations africaines orchestré entre 1964 et 1966 montrent que celles-ci sont capables de s'unir face aux dirigeants européens. Dans cette recherche d'unité, Djan occupe une place prépondérante. Il va en être de même sur un autre sujet de tension : l'exclusion de l'association d'Afrique du Sud de la FIFA pour des raisons de discrimination raciale.

\subsection{Entre politique et apolitisme. L'épineux cas de l'Afrique du Sud}

Au début des années 1950, la South African Soccer Federation (SASF), qui représente la majorité des clubs de la population noire commence à concurrencer la Football Association of Southern Africa (nom adopté en 1957 par l'association, FASA), qui se veut unitaire mais qui dans les faits est réservée exclusivement aux Blancs (Alegi \& Bolsmann, 2010). La FASA étant membre de la FIFA, la situation s'avère problématique lorsque la SASF demande son affiliation (Bolsmann, 2010, p. 36). Or la Fédération internationale n'accepte pas dans ses statuts la possibilité qu'un pays puisse disposer de deux associations membres. Après plusieurs années de discussions, et alors que la FASA reste la représentante légitime du football sud-africain à la FIFA, au début des années 1960, les militants sportifs sud-africains noirs protestent désormais de manière véhémente, et font appel à un mouvement de protestation transnational (Klotz, 1995 ; Cornelissen, 2011).

Fidèle à son appartenance au mouvement panafricain, Ohene Djan ne peut rester à l'écart des débats sur la situation en Afrique du Sud. Partant, il se solidarise avec l'ensemble des populations noires opprimées du continent. Cette lutte constitue un enjeu central de la politique internationale sportive du Ghana, et ce quels que soient les sports. De fait, Ohene Djan entretient rapidement des contacts étroits avec des sportifs sud-africains comme Dennis Brutus et George Singh qui

8 Ghana, Guinée, Cameroun, Soudan, Tunisie, Algérie, Liberia, Maroc, Sénégal, Mali, Éthiopie, Gabon, République arabe unie, Libye et Nigeria. 
- via des organisations comme le South African Non Racial Olympic Committee (SAN-ROC, créé en 1962) - militent en faveur des droits des sportifs de couleur en Afrique du Sud et dans les organismes sportifs internationaux. Très régulièrement, ils envoient des lettres ouvertes et des résumés sur la situation des sportifs noirs dans leur pays aux dirigeants sportifs des États africains, dont Djan'.

La première trace d'une opposition ghanéenne à la participation de l'Afrique du Sud aux joutes sportives peut être repérée dès 1959, lors d'une session du CIO à Rome (PRAAD, Procès-verbal de la réunion du Comité national olympique ghanéen du 7 octobre 1959). Au même moment, la question sud-africaine est débattue à chaque réunion du comité exécutif de la FIFA. En 1960, la FASA se voit poser un ultimatum d'un an pour abandonner la discrimination raciale sous peine d'être suspendue. Pour autant, une année plus tard, force est de constater qu'une majorité de l'élite dirigeante de la FIFA peine à prendre une véritable décision, ce qui pousse les dirigeants du football africain à penser que ses membres sont solidaires de la FASA (PRAAD, Lettre de Mourad Fahmy et Ydnekatchew Tessema aux membres du comité exécutif de la FIFA, 21 septembre 1961). À partir de cette date, les oppositions se radicalisent. Le Président de la FIFA, l'Anglais Stanley Rous, se fait le chantre de l'apolitisme en soulignant que la FIFA n'a pas à intervenir dans les affaires nationales d'un pays membre ${ }^{10}$. Mais, comme le souligne Peter Alegi, la situation est pour le moins ambiguë, car l'Anglais est historiquement proche des membres de la FASA - la FASA est d'ailleurs membre de la Football association jusqu'en 1962 - et dès lors n'est pas très enclin à favoriser une position antiraciste qu'il qualifie d'« agitation politique » et qu'il se refuse à considérer (2010, p. 74). En face de lui, les membres africains, dont Djan, décident dès lors de mener une campagne active en vue de l'exclusion de la FASA (PRAAD, Lettre de George Singh à Mourad Fahmy et Ohene Djan, 14 novembre 1963).

Dans une volonté de temporisation, la Fédération internationale envoie une commission d'enquête en 1963 pour statuer sur la question du non-respect de l'interdiction de la discrimination raciale par la FASA. Celle-ci est composée de Rous et d'un membre de son comité exécutif, Jimmy Maguire (Darby, 2003, p. 6). Mais la CAF désavoue cette mission qu'elle estime partiale et maintient sa demande d'exclusion de la FASA. Les blocs se figent. Rous perçoit l'opposition de la CAF à sa décision comme un acte de rébellion. Cela donne lieu à une discussion « vigoureuse et franche » entre lui et Djan lors d'une séance du comité exécutif de la FIFA qui se tient à Londres en septembre 1963 (PRAAD, Lettre d'Ohene Djan à Mourad Fahmy, 4 décembre 1963). L'échange se solde par une première victoire de Djan, puisqu'il obtient de ses collègues de reporter la discussion à l'assemblée générale ordinaire qui se déroulera l'année suivante à Tokyo. Il espère notamment qu'en assemblée plénière, l'intégration des nouvelles associations africaines lors de l'assemblée générale de 1964 fera pencher les votes en sa faveur.

Pendant la discussion, Rous et ses alliés accusent Djan d'être influencé par la conférence de l'OUA - qui s'est tenue six mois plus tôt à Addis-Abeba et durant laquelle Kwame Nkrumah prononce un discours radical dans lequel il condamne l'apartheid sud-africain (Daily Graphic, 25 mai 1963, pp. 2-7) - et donc de vouloir « faire entrer » la politique à la FIFA. Pour autant, lors des réunions du comité, Djan

9 Preuve de l'intérêt porté par Djan pour le lobbyisme du SAN-ROC, on retrouve plusieurs courriers et coupures de presse (sud-africaines - comme le Natal Mercury - ou non - comme l'East African Standard) sur le sujet dans les archives du département des sports du Ghana.

10 Ce n'est pas la première fois que la FIFA doit faire face à une telle situation. Au moment de la guerre d'Espagne, un cas relativement similaire s'était déclaré avec l'existence de deux fédérations. La Fédération internationale avait alors décidé de ne pas... choisir, attendant la fin du conflit avant de prendre une décision. Il s'agissait, selon son élite dirigeante, de ne pas interférer dans ce qu'ils considéraient alors comme une affaire interne à l'Espagne (Vonnard \& Quin, 2019). Au même moment que l'affaire sud-africaine, un autre cas problématique est également débattu, à savoir la reconnaissance simultanée de la Chine populaire et de Taiwan (Homburg, 2006). 
réfute l'hypothèse d'une influence des décisions de l'OUA sur sa position. Ce débat houleux donne lieu à deux échanges épistolaires parallèles. Dans une lettre à son collègue de la CAF, Mourad Fahmy, il analyse comme suit l'argumentaire qu'il a déployé face à Stanley Rous : " [Cette accusation ne peut] soutenir aucun test intelligent. [...] Il a été signalé que la lutte contre le racisme dans le football en Afrique du Sud était plus ancienne que l'historique conférence d'Addis-Abeba et que bien que les chefs d'États africains s'opposent politiquement au Portugal, les sportifs du continent ont accepté le Portugal parce qu'il pratique un sport multiracial " (PRAAD, Lettre d'Ohene Djan à Mourad Fahmy, 4 décembre 1963) .

Certes, comme le souligne le dirigeant ghanéen, d'une part l'ancienneté de la lutte anti-apartheid dans le domaine sportif africain est essentielle. D'autre part, les prises de position politiques de la CAF ne sont pas calquées sur celles de l'OUA. Cet argumentaire semble démontrer sa progressive maîtrise d'un des codes sociaux les plus importants de la FIFA, à savoir la rhétorique de l'apolitisme.

Parallèlement, Stanley Rous écrit à Djan pour poursuivre son argumentaire. Nous pouvons supposer que l'Anglais n'est pas dupe du raisonnement de Djan - celui-ci voit en lui un adversaire politique. Il essaie de déjouer la position du Ghanéen en lui adressant l'ultimatum suivant : "Nous devons éviter de mélanger la politique et le sport. Il doit n'y avoir absolument aucune interférence politique ou gouvernementale dans les affaires du football » (PRAAD, Lettre de Stanley Rous à la présidence de la CAF et à Ohene Djan, 15 novembre 1963).

En dépit de ce qui peut être considéré comme un rappel à l'ordre, Djan persiste. Une proposition d'expulsion de la FASA est finalement transmise à l'assemblée générale de la FIFA de 1964. Malgré un long plaidoyer de Stanley Rous, dans lequel il défend le rapport écrit quelques mois plus tôt, les congressistes suivent à une grande majorité (38 voix contre
15) l'argumentaire développé dans les discussions par son opposant ghanéen. Certes, la FASA n'est toujours pas exclue, Rous réussissant à ce qu'elle ne soit que suspendue, cependant il s'agit bien d'une victoire des dirigeants africains - et en particulier de Djan - qui ont su faire entendre leur voix.

Djan n'occupe certes pas les positions les plus importantes dans le comité exécutif de la FIFA. Mais, comme le montre l'exemple de son investissement dans la cause sud-africaine, il a néanmoins réussi à introduire au sein de l'organisation la vision panafricaine défendue par Kwame Nkrumah. Cette politisation par les acteurs africains va produire un contexte de tensions chroniques durant les années 1960-1970 au sein de la FIFA (Darby, 2002), et constitue un des éléments expliquant l'arrivée, à terme, d'un président non européen à la tête de l'organisation en 1974 : João Havelange (Sudgen \& Tomlinson, 1998; Darby, 2008)..

\section{ConCLUSION}

Djan n'aura toutefois pas le temps d'exercer bien longtemps ses fonctions au sein de la FIFA. En février 1966, quelques mois avant la Coupe du Monde en Angleterre, un coup d'État militaire renverse Kwame Nkrumah. Le Président s'exile chez son ami Sekou Touré, leader politique de la Guinée. Le National Liberation Council (NLC) qui prend le pouvoir écarte les fidèles du gouvernement. Dès lors, la junte militaire remplace immédiatement l'Osagyefo's Sportsman par un membre de son administration, William Marbell. La destitution ghanéenne de Djan a des effets très rapides à l'échelle internationale, Marbell devient le délégué de la Fédération nationale de football. De plus, il le désavoue de son poste au sein de la CAF et de la FIFA dès le printemps 1966 (FIFA, Lettre de Mourad Fahmy à Helmut Käser, 9 juillet 1966).

Au Ghana, Ohene Djan fait face à deux enquêtes publiques. L'une vise l'administration 
sportive et l'autre vise les malversations des hauts fonctionnaires. Elles conduisent à l'édition de deux rapports qui l'accablent : respectivement le rapport Tibo (1967) et le rapport Manyo-Plange (1969). Les deux commissions mettent en avant le train de vie luxueux de Djan, ses voyages coûteux et surtout sa proximité politique à Nkrumah. Très présent sur la scène médiatique de 1960 à 1966, il est une cible privilégiée de la nouvelle administration, qui veut couper avec l'ère nkrumahiste. Djan, évincé, se retire de la vie publique sportive, et son nom n'apparait plus que rarement aux côtés de clubs locaux jusqu'à son décès en 1987.

Au terme de cet article, nous pouvons souligner - en suivant les propos de Paul Darby (par exemple : Darby, 2019) - qu'Ohene Djan a incarné l'irruption d'un nouveau type de dirigeants sur la scène internationale. Entrer par la figure politisée de ce dirigeant sportif africain permet de mettre au jour les caractéristiques sociales qui définissent les dirigeants de la Fédération internationale durant les années 1950 et 1960. Ohene Djan, par son âge, sa nationalité et son engagement politique, se fond difficilement dans la masse des dirigeants européens qu'il rencontre en Europe. Mais ses compétences politiques et sociales, acquises en naviguant entre plusieurs mondes sociaux, lui permettent de décoder et de s'approprier les normes sociales et les sociabilités des dirigeants des associations européennes. Porté par la vague des indépendances qui secoue le monde, il prend part à une organisation pourtant très conservatrice et eurocentrée. Ainsi, les interactions d'Ohene Djan avec le monde de la FIFA nous donnent à voir tant le parcours d'un dirigeant sportif anticolonial que, en creux, le conservatisme et les pratiques politiques des dirigeants européens.

La focalisation sur Djan permet en outre de souligner des éléments liés à la politique internationale sportive du Ghana. Nous postulons que le travail de Djan de 1962 à 1966 s'apparente à une composante parmi d'autres de la diplomatie panafricaine du Ghana. En effet, son implication dans la lutte contre l'apartheid sud-africain et son combat pour l'obtention de places supplémentaires pour le continent africain au sein de la FIFA et dans la phase finale de la Coupe du monde donnent à voir le parcours d'un véritable ambassadeur sportif. Néanmoins, et il s'agit de toute la complexité de la diplomatie internationale sportive (Frank, 2012 ; Rofe, 2018, Rofe \& Tomlinson, 2019), Djan semble faire évoluer son mode de négociation au fur et à mesure de son implication croissante dans les affaires de la FIFA. Cette action souligne dès lors la prise d'importance des grandes organisations sportives et le fait qu'à partir de la seconde partie du $\mathrm{XX}^{\mathrm{e}}$ siècle, ces dernières commencent à peser sur la politique internationale des États et sur les manières d'agir de leurs représentants (Bernasconi, 2010 ; Vonnard \& Quin, 2017).

Enfin, la présente recherche appelle à de plus amples analyses sur les trajectoires et le rôle d'acteurs non européens et/ou non masculins au sein des organisations internationales sportives nées au cours du XX $\mathrm{X}^{\mathrm{e}}$ siècle (Cervin \& Nicolas, In Press). Ce type d'études permettra de préciser notre compréhension des codes et des normes qui gouvernent les relations sportives internationales et en particulier de nous aider à nous départir d'une vision sans doute encore trop eurocentrée de l'étude des organisations internationales sportives.

\section{SOURCES}

Procès-verbaux des réunions du comité exécutif et de l'assemblée générale de la FIFA. Archives de la Fédération internationale de football (FIFA).

Procès-verbaux de l'assemblée générale de la CAF. Archives de la Fédération internationale de football (FIFA).

Correspondance d'Ohene Djan et de la CAF. Archives nationales du Ghana (PRAAD).

Archives de la Central Organisation of Sports. Archives nationales du Ghana (PRAAD).

Manyo-Plange, J. S., Larbie, V. W. \& Quarshie, E. W., « Report of the Manyo-Plange (Assets) 
Commission ». Accra : National Liberation Council, 9 mai 1969. Archives nationales du Ghana (PRAAD).

Tibo, A., "The Tibo Committee Report on Ghana Sports ». Accra : National Liberation Council, 28 août 1967. Archives nationales du Ghana (PRAAD).

\section{BibLIOGRAPHIE}

Ahlman, J. S. (2017). Living with Nkrumahism : Nation, State, and Pan-Africanism in Ghana. Athens : Ohio University Press.

Alegi, P. (2010). African Soccerscapes : How a Continent Changed the World's Game. Athens : Ohio University Press.

Alegi, P. \& Bolsmann, C. (dir.). (2010). South Africa and the global game: football, apartheid and beyond. London : Routledge.

Allman, J. M. (1993). The Quills of the Porcupine : Asante Nationalism in an Emergent Ghana. Madison : University of Wisconsin Press.

Austin, D. (1966). Politics in Ghana, 1946-1960. LondonNew York : Oxford University Press.

Bayle, E. \& Clastres, P. (dir.). (2018). Global Sport Leaders: A Biographical Analysis of International Sport Management. Basingstoke: Palgrave Macmillan.

Bediako, K. (2012). The Complete History of the Ghana Football League, 1958-2012. Accra : self-published.

Bernasconi, G. (2010), De l'Universalisme au transnational : le Comité international olympique, acteur atypique des relations internationales. Bulletin de l'Institut Pierre Renouvin, 31(1), 151-159.

Bolsmann, C. (2010). White football in South Africa: empire, apartheid and change, 1892-1977. Soccer E Society, 11(1-2), 29-45.

Bouamama, S. (2014). Figures de la révolution africaine de Kenyatta à Sankara. Paris : La Découverte.

Cervin, G. \& Nicolas, C. (dir.). (Sous presse). Histories of Women's Work in Global Sport. A Man's World? Londres : Palgrave Macmillan.

Charroin, P. \& Waquet, A. (2008). L'universalisme professionalisant du football contre l'amateurisme internationaliste olympique. In T. Terret (dir.), Les Paris des Jeux olympiques de 1924 (pp. 445-490). Biarritz : Atlantica.

Cornelissen, S. (2011). Resolving " the South Africa problem »: Transnational activism, ideology and race in the Olympic movement, 1960-91. The International Journal of the History of Sport, 28 (1), 153-167.
Darby, P. (2000). Football, colonial doctrine and indigenous resistance : Mapping the political persona of FIFA's African constituency. Culture, Sport, Society, 3(1), 61-87.

Darby, P. (2002). Africa, football, and FIFA : politics, colonialism, and resistance. London-Portland : F. Cass.

Darby, P. (2003). Africa, the FIFA Presidency, and the Governance of World Football : 1974, 1998, and 2002. Africa Today, 50(1), 3-24.

Darby, P. (2008). Stanley Rous's 'Own Goal': Football Politics, South Africa and the Contest for the FIFA Presidency in 1974. Soccer E Society, 9 (2), 259-272.

Darby, P. (2013). "Let us rally around the flag ". Football, nation-building and pan-africanism in Kwame Nkrumah's Ghana. The Journal of African History, 54(2), 221-246.

Darby, P. (2019). Politics, resistance and Patronage: the African Boycott of the 1966 World Cup and its Ramification. Soccer E Society.

Defrance, J. (2000). La politique de l'apolitisme. Sur l'autonomisation du champ sportif. Politix. Revue des sciences sociales du politique, 13(50), 13-27.

Dietschy, P. (2011). French Sport: Caught between Universalism and Exceptionalism. European Review, 19 (4), 509-525.

Dietschy, P. (2013). Making football global ? FIFA, Europe, and the non-European football world, 1912-74. Journal of Global History, 8(2), 279-298.

Dietschy, P. \& Kemo-Keimbou, D.-C. (2008). L'Afrique et la planète football. Luçon : Pollina Imprimerie.

Dullin, S. (2001). Des hommes dinfluences. Les ambassadeurs de Staline en Europe, 1930-1939. Paris : Payot.

Duschesne, V. \& Guedj, P. (2005). «Akonedi ne voyage pas » : la formation d'un réseau transnational akan entre le Ghana et les États-Unis. In L. Fourchard, A. Mary \& R. Otayek (dir.). Entreprises religieuses transnationales en Afrique de l'Ouest (pp. 135-153). Paris : Karthala.

Eisenberg, C., Lanfranchi, P., Mason, T. \& Wahl, A. (2004). FIFA 1904-2004. Le siècle du football. Paris : Le Cherche Midi.

Frank R. (2012). Internationalisation du sport et diplomatie sportive. In R. Frank (dir.), Pour l'histoire des relations internationales (pp. 387-405). Paris : Presses universitaires de France.

Homburg, H. (2006). FIFA and the "Chinese Question ", 1954-1980 : an exercise of statutes. Historical Social Research, 31(1), 69-72.

Klotz, A. (1995). Norms in International Relations : the Struggle against Apartheid. New York : Cornell University Press. 
Louis, M. (2017). Une représentation dépolitisée ? L'Organisation internationale du travail de 1919 à nos jours. Critique internationale, 76(3), 61-80.

Macon, B. (2008). The Politization of football : the European Game and the Approach to the Second World War. Soccer E Society, 9(4), 532-555.

Miescher, S. (2005). Making men in Ghana. Bloomington : Indiana University Press.

Mittag, J. (2015), Negociating the Cold War ? Perspectives in Memory Research on UEFA, the Early European Football Competitions and the European Nations Cups. In W. Pyta \& N. Havemann (dir.), European Football and Collective Memory (pp. 40-63). Basingstoke : Palgrave Macmillan.

Monfils, B. S. (1977). A Multifaceted Image : Kwame Nkrumah's Extrinsic Rhetorical Strategies. Journal of Black Studies, 7(3), 313-330.

Newell, S. (2006). Entering the Territory of Elites : Literary Activity in Colonial Ghana. In K. Barber (dir.), Africa's hidden histories : everyday literacy and making the self (pp. 211-235). Bloomington : Indiana University Press.

Nicolas, C. (2017). Des corps connectés : les Ghana Young Pioneers, tête de proue de la mondialisation du nkrumahisme (1960-1966). Politique africaine, 147(3), 87-107.

Parks, J. (2014). Welcoming the "Third World » : Soviet Sport Diplomacy, Developing Nations and the Olympic Games. In H. Dichter \& A. Johns (dir.), Diplomatic Games: Sport, Statecraft, and International Relations since 1945 (pp. 85-114). Lexington Kentucky : University Press of Kentucky.

Plageman, N. (2013). Highlife Saturday night: popular music and social change in urban Ghana. Bloomington : Indiana University Press.
Quin, G., Vonnard, P. \& Jaccoud, C. (dir.) (sous presse). Des hommes et des réseaux. Le rôle de la Suisse dans l'internationalisation du sport (1912-1972). Neuchâtel : Alphil-Presses universitaires suisses.

Rofe, S. J. (dir.). (2018). Sport and Diplomacy. Games within games. Manchester : Manchester University.

Rofe, S. J. \& Tomlinson, A. (2019). The Untold Story of FIFA's Diplomacy and the 1966 World Cup: North Korea, Africa and Sir Stanley Rous. The International History Review.

Sudgen, J. \& Tomlinson, A. (1998). FIFA and the Contest for World Football: Who Rules the People's Game? Cambridge: Polity press.

Tomlinson, A. (2000). FIFA and the men who made it. Soccer E Society, 1(1), 55-71.

Vieta, K. T. (1999). Flagbearers of Ghana. Accra : ENA Publication.

Vonnard, P. (2017). Euvrer en faveur du football européen. Jalons biographiques sur les précurseurs de l'UEFA (1920-1950). In W. Gasparini (dir.), L'Europe du football. Socio-histoire d'une construction européenne (pp. 107-121). Strasbourg : Presses universitaires de Strasbourg.

Vonnard, P. (2018). L'Europe dans le monde du football. Genèse et formation de l'UEFA (1930-1960). Bruxelles : Peter Lang.

Vonnard, P. \& Quin, G. (2017). Studying International Sports Organisations during the Cold War. Sport History, 37(3), 265-272.

Vonnard, P. \& Quin, G. (2019). Promouvoir et jouer au football pendant la guerre. La Fédération internationale de football association, les forces de l'Axe et la Deuxième Guerre mondiale. Hispania Nova, 17, 306-340.

\section{Resumen : Ohene Djan. ¿Un activista panafricanista para conquistar la FIFA?}

En 2004, el Estadio Nacional de Accra pasó a llamarse Estadio Ohene Djan, conmemorando el trabajo del primer director de deportes en Ghana (1960-1966). Sin embargo, seis años después, el municipio de Accra reconsidera esta elección de las autoridades nacionales, argumentando que es un estadio municipal y no un estadio nacional justificando esta decisión. Si se habla hoy todavía de Ohene Djan, es porque su acción probablemente ha ido mucho más allá de las fronteras de Ghana. De hecho, Kwame Nkrumah fue nombrado director de deportes en Ghana en 1960. Durante los seis años de su mandato, Kwame Nkrumah encarnó el surgimiento de apuestas políticas en el fútbol africano y trabajó para reconfigurar las relaciones diplomáticas deportivas hasta ahora en gran parte confinado a Europa y Sudamérica.

Como parte de esta contribución, se trata de pensar en la biografía de Djan como un punto de encuentro entre el fútbol -nacionales y mundiales, por un lado, y entre una trayectoria política y deportiva por el otro. Menos que la narrativa de la cronología de un viaje biográfico por etapas organizativas, políticas, personales y deportivas, este artículo considera a Djan como el núcleo que nos permite cuestionar el modelo deportivo y político de la FIFA, afirmando ser apolítico y élitivo. 
Esta investigación se basa en la documentación recopilada en importantes archivos de la Federación Internacional de la Asociación de Fútbol (FIFA) que ha sido cruzada por información de los fondos de Archivos Nacionales de Ghana. Finalmente, el estudio se beneficia de la lectura de los deportes de Ghana y de los principales periódicos, que destacan elementos que no se abordan en los documentos oficiales de la FIFA o en los archivos nacionales de Ghana, sino también poder mirar la presencia pública de Djan.

Palabras clave: Historia, fútbol, Ghana, FIFA, organizaciones internacionales, Panafricanismo

\section{Zusammenfassung: Ohene Djan. Ein panafrikanischer Kämpfer bei der Eroberung der FIFA?}

Im Jahr 2004 wurde das Nationalstadion von Accra in Ohene Djan Stadion umbenannt, um an die Arbeit des ersten Sportdirektors Ghanas (1960-1966) zu erinnern. Sechs Jahre später jedoch machte die Gemeinde Accra diese Namensgebung der nationalen Behörden rückgängig und argumentierte zur Rechtfertigung dieser, dass es sich um ein städtisches Stadion und kein Nationalstadion handele. Wenn Ohene Djan auch heute noch für Schlagzeilen sorgt, dann wahrscheinlich, weil sich sein Handeln weit über die Grenzen Ghanas hinaus erstreckt hat. 1960 von Kwame Nkrumah zum Sportdirektor Ghanas ernannt, verkörperte er während seiner sechsjährigen Amtszeit die Debatte einzigartiger afrikanischer politischer Themen im Weltfußball und arbeitete an der Neuordnung diplomatischer Sportbeziehungen, die bisher weitgehend auf Europa und Südamerika beschränkt waren.

Im Rahmen dieses Beitrags geht es darum, über Djans Biografie als Schnittpunkt zwischen dem Fußball - national und global - auf der einen Seite und einer politischen und sportlichen Entwicklung auf der anderen Seite nachzudenken. Der Artikel betrachtet Djan hier weniger aus der Sicht einer biographischen Chronologie, die von organisatorischen, politischen, persönlichen und sportlichen Etappen unterbrochen wird, sondern als ein Bindeglied, das es uns erlaubt, das sportliche und politische Modell der FIFA in Frage zu stellen, das angeblich unpolitisch und elitär ist.

Diese Forschung basiert auf Dokumenten aus dem Archiv der Fédération internationale de football association (FIFA), die mit Informationen aus dem Bestand des Nationalarchivs von Ghana verglichen wurden. Schließlich profitiert die Studie von der Lektüre ghanaischer Zeitungen und Sportzeitungen, die Aspekte hervorheben, die nicht in offiziellen FIFA-Dokumenten oder in Ghanas Nationalarchiv enthalten sind, aber auch einen Einblick in die öffentliche Präsenz von Djan geben.

SCHLaGWÖRTER: Geschichte, Fußball, Ghana, FIFA, internationale Organisationen, Panafrikanismus

\section{Riassunto : Ohene Djan. Un militante africanista alla conquista della FIFA ?}

Nel 2004, lo stadio nazionale di Accra è rinominato Ohene Djan, questo gesto doveva permettere di commemorare l'azione del primo direttore degli sport del Ghana (1960-1966). Argomentando tuttavia che si tratta di uno stadio municipale e non di uno stadio nazionale per giustificare questa decisione. Se Ohene Djan fa ancora parlare ai nostri giorni, ciò è dovuto senza dubbio al fatto che la sua azione ha largamente superato le frontiere del Ghana. In effetti, nominato direttore degli sport del Ghana nel 1960 da Kwame Nkrumah, quest'ultimo ha incarnato durante i sei anni del suo mandato l'irruzione di poste in gioco politiche propriamente africane in seno al football mondiale, ed ha operato a riconfigurare delle relazioni diplomatiche sportive fino a quel momento limitate all'Europa e all'America del Sud.

Nel quadro di questo contributo, si tratta di pensare il percorso biografico di Djan come un punto d'incontro tra i footbal - nazionale e globale - da una parte e tra una traiettoria politica e sportiva dall'altra parte. Meno che il racconto della cronologia di un percorso biografico ritmato da tappe organizzazionali, politiche, personali e sportive, questo articolo considera Djan come il nodo che 
ci permette d'interrogare il modello sportivo e politico della FIFA, rivendicato come apolitico ed elitario.

La presente ricerca si appoggia su una documentazione raccolta nei ricchi archivi della Fédération internationale de football association (FIFA) che è stata incrociata con informazioni uscite dai fondi degli Archivi nazionali del Ghana. Infine, lo studio beneficia della lettura di giornali sportivi e generalisti ghanesi, che permettono di mettere in luce degli elementi che non sono trattati nei documenti ufficiali della FIFA o negli archivi nazionali ghanesi, ma anche di avere uno sguardo sulla presenza pubblica di Djan.

Parole Chiave : FIFA, football, Ghana, organizzazioni internazionali, Panafricanismo, storia. 